\title{
Estabilidad De Voltaje En Redes De Distribución Eléctrica Monofásicas De Medio Voltaje, Aplicando Reguladores Quick Drive Tap En Estado Estable
}

Voltage Stability in Medium Voltage Single-Phase Electrical Distribution Networks, Applying Quick Drive Tap Regulators in Steady State

\begin{tabular}{c} 
Cárdenas D. ${ }^{1}$, Chávez C. ${ }^{2}$ y Layedra N. ${ }^{2}$ \\
$\begin{array}{c}{ }^{1} \text { Universidad Politécnica Salesiana, Carrera de Telecomunicaciones, Guayaquil-Ecuador } \\
\text { e-mail: dcardenasv@ups.edu.ec }\end{array}$ \\
$\begin{array}{c}{ }^{2} \text { Universidad Politécnica Salesiana, Carrera de Electricidad, Guayaquil-Ecuador } \\
\text { e-mail: cchavez@ups.edu.ec, e-mail: nlayedra@ups.edu.ec }\end{array}$ \\
\hline $\begin{array}{c}\text { Información del artículo } \\
\text { Recibido: } 15 / 01 / 2021\end{array}$ \\
Aceptado: $17 / 03 / 2021$ \\
\hline
\end{tabular}

\section{RESUMEN}

En este trabajo se propone desarrollar un análisis técnico de una red de distribución monofásica radial en media tensión de un sector del cantón Daule de la Corporación Nacional de Electricidad Unidad de Negocio Guayas Los Ríos, CNEL EP GLR; con el fin de mejorar los perfiles de tensión de la red. Se analizaron las causas que provocan el colapso del voltaje, y los medios para mitigar este problema. El método utilizado para mantener la estabilidad del voltaje en la red, motivo de este estudio, es utilizando reguladores de tensión Quick Drive Tap, y se establece mediante simulación en el software CYME, en el cual se desarrolló el análisis técnico en estado estable, con varios escenarios para encontrar la localización óptima y el dimensionamiento de los reguladores. Finalmente, se desarrolla una comparación de la red de distribución antes y después de lograr que los niveles de voltaje estén dentro de los porcentajes admitidos por el ente regulador de las empresas eléctricas de distribución, ARCONEL.

Palabras clave: Reguladores de Voltaje, CYME, ARCONEL, CNEL

\begin{abstract}
This document proposes to develop a technical analysis of a medium voltage single-phase radial distribution network in a sector of the Daule canton of the CNEL EP GLR; in order to improve network voltage profiles. Moreover the causes that induce the voltage collapse and the means to mitigate this problem, were analyzed. The method used to maintain the stability of the voltage in the network, the reason for this study, is using Quick Drive Tap voltage regulators, and it is setting up by simulations in the CYME software, in which the technical analysis in steady state were developed, with several scenarios using the heuristic method, to find the optimal location and sizing of the regulators. To conclude, it will be setting a comparison of the before and after the distribution network achieves the voltage levels that are within the percentages admitted by the regulatory institution of the electrical distribution companies, ARCONEL.
\end{abstract}

Keywords: Voltage Regulators, CYME, ARCONEL, CNEL 


\section{Introducción}

Las empresas eléctricas de distribución tienen como objetivo principal, suministrar el servicio público de energía eléctrica para satisfacer las necesidades del consumidor final, de una manera confiable, segura y cumpliendo con los estándares de calidad. Sin embargo, en el momento de que los sistemas de distribución entran en operación se generan efectos no deseados, tales como la caída del voltaje de suministro, lo cual ocasiona pérdidas significativas en el usuario final.

En consecuencia, a este efecto inevitable de caída del voltaje, las empresas eléctricas de distribución se han visto forzadas a mejorar su infraestructura, con el fin de entregar un buen producto al consumidor final y poder cumplir con sus objetivos.

A pesar de los esfuerzos realizados por la mejora del voltaje a través del balanceo de fases, instalación de banco de capacitores, reemplazo de conductor, etc., esto no ha sido posible en ciertos sectores; como es en el caso de la Corporación Nacional de Electricidad Unidad de Negocio Guayas Los Ríos, CNEL EP GLR, en donde existe un sector con mayor problema de estabilidad del voltaje que se encuentra en el cantón Daule, debido a que es un sector agrícola y contiene una gran cantidad de piladoras de granos.

En este documento se analizará puntualmente el comportamiento de un alimentador rural de media tensión El Limonal de la Subestación Daule Norte de 2,8MVA, frente a la solución de caída de voltaje por medio de reguladores de tensión Quick Drive Tap.

\section{Método}

\section{Sistema de distribución de energía eléctrica}

\section{A. Aspectos generales}

La red de distribución del suministro de energía eléctrica es una parte muy importante de los sistemas de potencia (Generación, Transmisión y Distribución), cuyo fin es el de entregar energía eléctrica desde la subestación de distribución hasta el consumidor final, los cuales se encuentran dispersos en grandes territorios y con cargas de diversas magnitudes. Los elementos que componen los sistemas de distribución son: Líneas de Subtransmi- sión, Subestaciones de Distribución, Alimentadores Primarios, Transformadores de Distribución y Red Secundaria [6].

\section{B. Normativa de calidad del servicio eléctrico de distribución en el ecuador}

Una de las obligaciones de las Empresas Eléctricas de Distribución del Ecuador es cumplir con entregar a los clientes un nivel de voltaje que se encuentre dentro de los límites establecidos por la Resolución Nro. ARCONEL 053/18 de la Regulación 005/18 «Calidad del servicio de distribución y comercialización de energía eléctrica»; en donde se realiza la evaluación de la calidad del suministro del servicio eléctrico, tomando en cuenta los siguientes parámetros [2]:

\section{Indice de calidad del producto}

Se representa principalmente por el nivel de voltaje, las perturbaciones que se presentan en el voltaje de manera transitoria (Flicker), la distorsión de frecuencias armónicas en la señal de voltaje, y el desequilibrio de los niveles de tensión [7].

\section{2.Índice de calidad del servicio técnico}

Se representa por la cantidad de interrupciones (FMIK) y persistencia de las interrupciones (TTIK) a nivel general y por usuario:

$$
\begin{aligned}
& F M I K=\frac{\sum_{i} k V A_{i}}{k V A_{\tau}} \\
& T T I K=\frac{\sum_{i} k V A_{i} \cdot t_{i}}{k V A_{\tau}}
\end{aligned}
$$

Donde:

FMIK = Frecuencia promedio de las interrupciones

TTIK = Tiempo promedio de interrupciones

$k V A_{i}=$ Potencia aparente en kVA sin servicio eléctrico por la interrupción $i$

$k V A_{\tau}=$ Potencia aparente en kVA nominal instalados en el alimentador

$t_{i}=$ Tiempo de la interrupción $_{i}$ 


\section{Indice de calidad del servicio comercial}

Se representa como la tasa de atención a nuevos usuarios, tasa de falla en la facturación, tasa e índice de tiempo de resolución de los problemas, índice de reposición del servicio a los usuarios; tasa de contestación a las consultas de los clientes; nivel de satisfacción de los usuarios.

Para fines de estudio de este documento, nos enfocaremos en el nivel de voltaje, correspondiente al ítem A «Calidad del Producto».

El índice de la calidad de nivel de voltaje en un punto del sistema de distribución, según la regulación del ARCONEL, se determina con la siguiente fórmula [2]:

$$
\Delta V_{k}=\frac{V_{k}-V_{N}}{V_{N}} \times 100[\%]
$$

Donde:

$$
\begin{aligned}
\Delta V_{k}= & \begin{array}{l}
\text { Cambio del voltaje de suministro } \\
\text { en comparación al voltaje nominal } \\
\text { en el punto } k .
\end{array} \\
V_{k}= & \begin{array}{l}
\text { Tensión de suministro en el punto } \\
k, \text { definido como el valor medio de }
\end{array} \\
\text { las medidas registradas. } & \text { Tensión nominal en el punto } k .
\end{aligned}
$$

Tabla 1. Límites exigidos por la regulación del Arconel sobre variaciones de voltaje

\begin{tabular}{lll}
\hline Nivel de Voltaje & & Rango admisible \\
\hline Alto Voltaje Grupo 1 & $>138 \mathrm{kV}$ & $\pm 5.0 \%$ \\
& $>40 \mathrm{kV}$ y & $\pm 5.0 \%$ \\
Alto Voltaje Grupo 2 & $\leq 138 \mathrm{kV}$ & \\
& $>0,6 \mathrm{kV}$ y & $\pm 6.0 \%$ \\
Medio Voltaje & $\leq 40 \mathrm{kV}$ & \\
& $\leq 0,6 \mathrm{kV}$ & $\pm 8.0 \%$ \\
\hline
\end{tabular}

\section{Resultados y discusión.}

\section{Problemas de bajos niveles de voltaje y po- sibles soluciones}

En los componentes eléctricos de las líneas de distribución se producen caídas de voltajes que deben diagnosticarse con mucho detalle, con el objetivo de que, los cambios normales de carga, la tensión se permanezca dentro de los rangos es- tablecidos como normales. Un voltaje bajo puede ocasionar problemas en los equipos ya sean de uso industrial (motores), comercial o residencial (electrodomésticos), lo cual genera un gasto económico importante debido a la necesidad de reparar o adquirir equipos nuevos [8].

Algunas causas más frecuentes que producen la caída de tensión y sus respectivos efectos asociados en las redes de distribución eléctrica son:

\section{Sobrecarga o saturación del sistema eléctrico}

La sobrecarga de las líneas de distribución se presenta cuando la capacidad de la demanda excede el valor del diseño de las líneas, lo cual es muy común en zonas con temperaturas elevadas, que en temporadas de calor se da la necesidad del uso del aire acondicionado y esto genera un incremento de demanda [3] [9].

\section{Largas distancias de líneas de distribución}

El problema de bajo voltaje es común en líneas de distribución que realizan grandes recorridos desde la cabecera del alimentador (en la subestación de distribución) hasta el usuario final. La resistencia del conductor se incrementa en función del aumento de la longitud de la línea de distribución.

\section{Calibre del conductor}

El calibre de conductor también infiere en la distorsión del voltaje debido a que la resistencia del conductor varía de acuerdo con su calibre. A mayor calibre de conductor, es menor su resistencia.

Para mitigar los problemas presentados de bajo voltaje a nivel de distribución existen varias opciones [10], lo cual es recomendable realizar un análisis técnico-económico ya que se puede incurrir en gastos con montos elevados y con poca mejoría. Entre las soluciones existentes, tenemos [11]:

- Construir una subestación cerca de la mayor concentración de la carga con nuevos alimentadores primarios.

- Uso de cambiadores de tomas del transformador de poder de la subestación (TAP).

- Uso de cambiadores de tomas de los transformadores de distribución. 
- Instalación de bancos de capacitores.

- Instalación de reguladores de voltaje.

- Equilibrio de distribución de cargas en las troncales de los alimentadores.

- Aumento del calibre de los conductores.

- Transfiriendo cargas a nuevos alimentadores.

- Distribuir a un nivel de tensión mayor a $13.8 \mathrm{kV}$, como es el caso de algunas empresas eléctricas del ecuador, que se está distribuyendo a un nivel de tensión de $22 \mathrm{kV}$.

El tipo de solución a implementar depende de las características y particularidades del sistema eléctrico [4]. No obstante, en los métodos de regulación automáticos, el control de la tensión requiere actuar en tres niveles:

1. Regulación del nivel de voltaje los puntos de entrega de las subestaciones de distribució

2. Regulación mediante la instalación de equipos en el arranque del alimentador de la subestación.

3. Regulación mediante la instalación de equipos a lo largo del alimentador.

Las subestaciones del sistema de eléctrico de distribución están equipadas con transformadores de potencia que incluyen conmutadores de taps que operan automáticamente bajo carga. Como respaldo, en algunas subestaciones se puede tener la opción de reguladores de voltajes que actúan de manera independiente por cada alimentador.

Los equipos de regulación de voltaje están diseñados para mantener de manera automática el nivel establecido de tensión, aún sobre los cambios bruscos de carga. Es decir, cuando la carga aumenta, el trabajo del regulador es elevar el voltaje en el alimentador para compensar la caída de tensión en el alimentador del sistema de distribución. Cuando los alimentadores de distribución tienen longitudes muy extensas y los consumidores están ubicados en zonas muy alejadas, se puede requerir la instalación de capacitores o reguladores de voltajes en puntos específicos del alimentador, como un recurso de ayuda complementaria para una regulación más precisa [5].

Desde el punto de vista técnico y financiero se sugiere el uso de reguladores y condensadores para mejorar las condiciones de los niveles de voltaje.
En este caso, para el análisis del comportamiento del voltaje, se aplicarán reguladores automáticos.

El uso de los reguladores es muy extendido en los puntos de utilización, es decir, en las zonas de los usuarios, con el objetivo de mantener constante el voltaje que suministran los alimentadores individualmente. Los reguladores de voltaje pueden ser de tipo de inducción o de tipo escalón. Los de tipo escalón o discretos pueden ser de tipo estación monofásicos y trifásicos, los cuales pueden usarse en subestaciones para la regulación de las barras o para la regulación del voltaje tanto en la cabecera como a lo largo de los alimentadores.

Los reguladores de voltaje tipo escalón, son autotransformadores que presentas varias derivaciones (taps) en las bobinas, en conexión serie, con porcentajes definidos de incremento o decremento del $10 \%$ con respecto al nivel de voltaje nominal, en 32 conmutaciones, con 5/8\% de variación de tensión por paso [1].

Un regulador de voltaje se compone de dos partes: equipo de fuerza y equipo de control. El equipo de fuerza, además del autotransformador contiene los equipos de medición (transformadores de voltaje y de corriente). El equipo de control tiene entradas de los equipos de medición lo cual le permite tener un control de nivel de voltaje y del rango (ancho de banda) [12].

\section{Software de simulación de sistemas de dis- tribución CYME}

Es un software de análisis de sistemas eléctricos de distribución que consta de varias aplicaciones para conformar un editor de redes, con bibliotecas de modelos que el usuario puede personalizar con el objetivo de obtener soluciones eficientes en el comportamiento de las redes eléctricas [13].

Los módulos que incluye este software contienen una serie de herramientas avanzadas y bibliotecas con amplia información actualizada para el estudio del comportamiento de sistemas industriales, de transmisión o distribución de la energía eléctrica [13].

La ventaja principal del uso de éste software para nuestra investigación es que nos permite efectuar 
algunos tipos de análisis en redes equilibradas o desequilibradas, monofásicas, bifásicas o trifásicas, de forma radial, en anillo o en malla [13].

Para la lograr la estabilidad del voltaje, el software aplica las siguientes técnicas: la reconfiguración de redes, el equilibrado o balance de fases, la ubicación óptima de los condensadores shunt y de los reguladores de tensión en la red [13].

Los problemas de estabilidad de voltaje presentan situaciones muy complejas en el ámbito de la ingeniería tales como determinar la ubicación y cantidad de reguladores, con el fin de solucionar los problemas de estabilidad con la consecuencia de disminuir los costos de energía [13].

\section{Ubicación óptima de reguladores de voltaje Quick Drive Tap en estado estable}

La estabilidad del voltaje en una red de media tensión monofásica se la realizará con los reguladores de voltaje Quick Drive Tap, analizando el comportamiento del resultado final en estado estable. En primer lugar, elegiremos el lugar óptimo para instalar el regulador de voltaje a través de un método heurístico y con la ayuda del software de simulación de sistemas de potencia CYME 7.1 Rev 02. Posteriormente se calculará la capacidad del regulador en base a la ubicación elegida y carga que alimentará, y finalmente se realizará el análisis con los resultados obtenidos.

Para este análisis se toma como ejemplo el Alimentador Limonal de la Subestación Daule Norte perteneciente a la Empresa Eléctrica de Distribución CNEL EP UNIDAD DE NEGOCIO GUAYAS LOS RIOS. Según los históricos de carga y de voltaje, éste alimentador presenta problemas de bajo voltaje el cual se intensifica en los ramales monofásicos.

1.Características técnicas y condiciones iniciales del Alimentador El Limonal de la Subestación Daule Norte

El Alimentador Limonal sirve de energía continua a los clientes residenciales, comerciales e industriales de la Parroquia Limonal. En la figura 1 se observa el recorrido del alimentador primario con sus respectivos ramales de manera geo referencial. La línea de color verde corresponde a la troncal del alimentador, y la línea de color azul corresponde al ramal monofásico motivo de análisis.

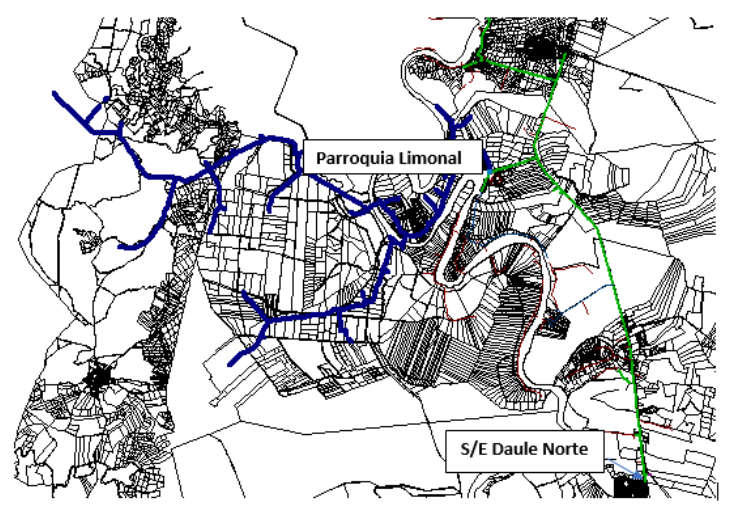

Figura 1. Topología del alimentador Limonal de la S/E Daule Norte. La Troncal del Alimentador de color verde y El Ramal de estudio de color azul. Software

CYME

El alimentador de media tensión Limonal tiene una longitud de $9,61 \mathrm{~km}$ con calibre de conductor ACSR 4/0, cuenta con $54,31 \mathrm{~km}$ de línea monofásica y $9,74 \mathrm{~km}$ de línea bifásica.

El alimentador El Limonal suministra continuamente energía eléctrica a 4376 abonados y registra una potencia aparente máxima de 2,8 MVA en el mes de agosto de 2020 con 5904 KVA conectados a lo largo de la red de media tensión.

En la figura 2 se muestra el histórico por mes de la potencia aparente máxima registrada en el alimentador El Limonal verificando que la cargabilidad máxima en el año 2019 llego al 40\% con respecto a su capacidad nominal en temporada de invierno.

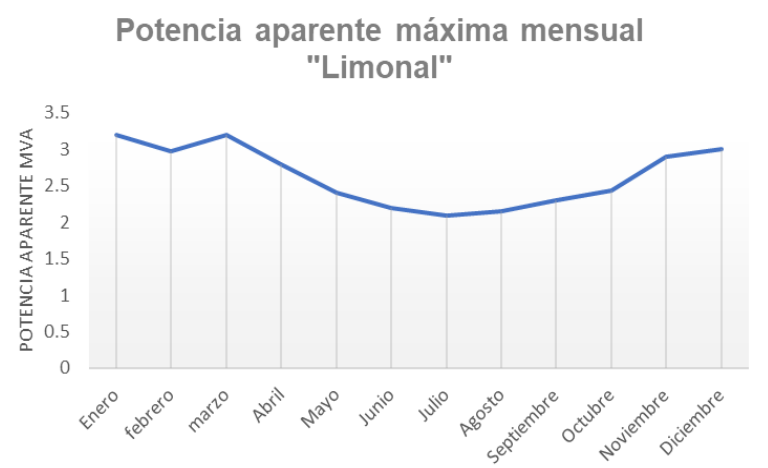

Figura 2. Historial por mes del año 2019 de la Potencia aparente del alimentador Limonal. Software CYME 
En la figura 3 se observan los indicadores de calidad de servicio técnico FMIK y TTIK del alimentador El Limonal, los cuales demuestran los problemas existentes en el alimentador debido a las interrupciones. El peor escenario se observa en los meses de mayo y junio del año 2020, donde los usuarios presentaron 7 interrupciones de servicio y un tiempo de afectación total de 8,25 horas.

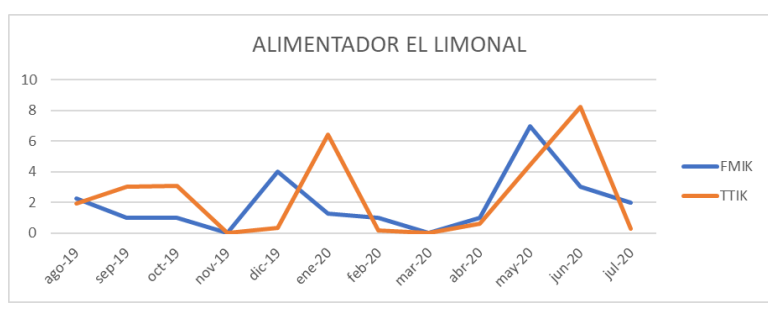

Figura 3. Indicadores de calidad de servicio técnicos del alimentador Limonal. Software CYME

Nos enfocamos en la línea de media tensión «monofásica» con mayores usuarios conectados y con mayor carga conectada, el cual se registra 381 usuarios conectados al ramal con calibre ACSR 2 con longitud de $8,67 \mathrm{~km}$.

En el mes de junio de 2019 se intentó mejorar los niveles de voltaje en el sector con el ingreso de una subestación móvil cercana a la mayor concentración de carga. La simulación se la realizará con los registros de los últimos meses del año tanto en máxima como en mínima demanda para verificar los parámetros eléctricos (voltaje, corriente, fp, potencias) actuales que se están presentando en el ramal monofásico motivo de análisis.

En la tabla 2, se muestra el resultado del flujo de potencia en demanda máxima del ramal monofásico que suministra energía eléctrica al cantón Limonal, verificando que su demanda máxima es 131,8 KVA.

Tabla 2. Resultado del flujo de potencia en demanda máxima de ramal monofásico.

\begin{tabular}{llllllll}
\hline FASE & kVLL & kVLN & $\mathbf{i}(\mathbf{A})$ & kVA & kW & kVAR & FP \\
\hline A & & 7,353 & 17,932 & 131,838 & 117,69 & $-59,41$ & $-89,271$ \\
& Ineutro & 5,977 & Total: & 131,838 & 117,69 & $-59,41$ & \\
\hline
\end{tabular}

En la figura 4, se muestra el perfil de voltaje en demanda máxima del ramal monofásico de $8,67 \mathrm{~km}$ visto desde el alimentador de cabecera hasta el último tramo de línea.

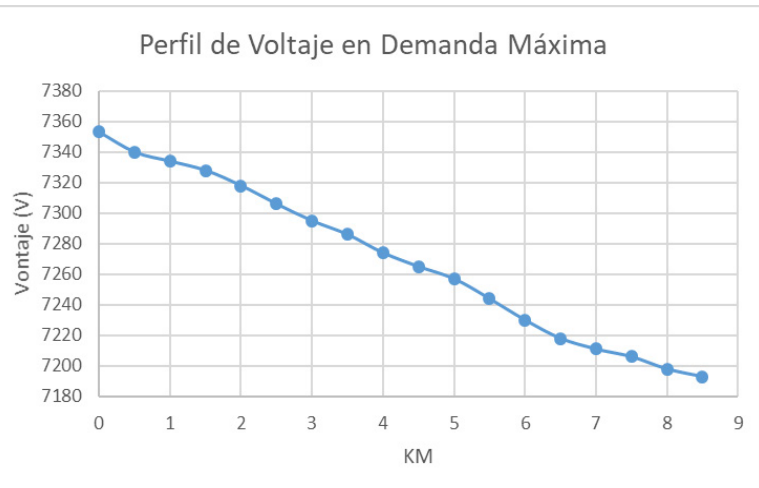

Figura 4. Perfil de voltaje en demanda máxima del ramal monofásico. Software CYME

Se verifica que los voltajes en demanda máxima a lo largo de la línea monofásica se encuentran en el rango de 7353-7193 (V) lo cual resulta un porcentaje de variación de voltaje en promedio de $-8,71 \%$ que se excede del límite del $\pm 6 \%$ estipulado en la regulación del ARCONEL 005/18, entregando, así, una mala calidad de servicio eléctrico a los usuarios finales e incumpliendo con la regulación.

En la tabla 3, se muestra el resultado del flujo de potencia en demanda mínima del ramal monofásico que suministra energía eléctrica al cantón Limonal, verificando que su demanda mínima es 92,3 KVA.

Tabla 3. Resultado del flujo de potencia en demanda mínima de ramal monofásico

\begin{tabular}{llllllll}
\hline FASE & kVLL & kVLN & $\mathbf{i}(\mathbf{A})$ & kVA & kW & kVAR & FP \\
\hline \multirow{2}{*}{$\mathrm{A}$} & & 7,639 & 12,089 & 92,338 & 80,109 & $-45,921$ & $-86,757$ \\
& \multirow{2}{*}{ Ineutro } & 4,03 & Total: & 92,338 & 80,109 & $-45,921$ & $-86,757$ \\
\hline
\end{tabular}

En la figura 5, se muestra el perfil de voltaje en demanda mínima del ramal monofásico de $8,67 \mathrm{~km}$ visto del arranque hasta el último tramo de línea. 


\section{Perfil de Voltaje en Demanda Mínima}

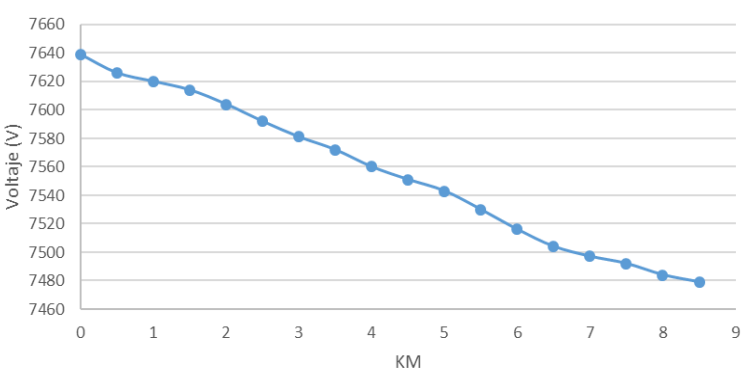

Figura 5. Perfil de voltaje en demanda mínima del ramal monofásico. Software CYME

Se verifica que los voltajes en demanda mínima a lo largo del ramal monofásico se encuentran en el rango de 7639-7479 (V) entregando un nivel de voltaje muy cercano a los límites establecidos por la regulación 005/19.

Se verifica que los voltajes en demanda mínima a lo largo del ramal monofásico se encuentran en el rango de 7639-7479 (V) lo cual resulta un porcentaje de variación de voltaje en promedio de $-5,12 \%$ que está dentro del límite del $\pm 6 \%$ estipulado en la regulación del ARCONEL $005 / 18$, entregando así, una calidad de servicio eléctrico aceptable y sin incurrir en sanciones a la distribuidora.

\section{Mejora de los niveles de voltaje del Alimentador El} Limonal de la Subestación Daule Norte

Para mejorar el voltaje en el ramal monofásico por caídas de voltaje desde la Subestación de Distribución Daule Norte se instala un regulador monofásico automático en las coordenadas que se observa en la figura 6 por ser el nodo que contiene la mayor concentración de carga y el más afectado por la caída de voltaje. Se procede a realizar un flujo de carga tanto en mínima y máxima demanda, a través del software CYME, para verificar el cumplimiento de la regulación del ARCONEL 005/18.

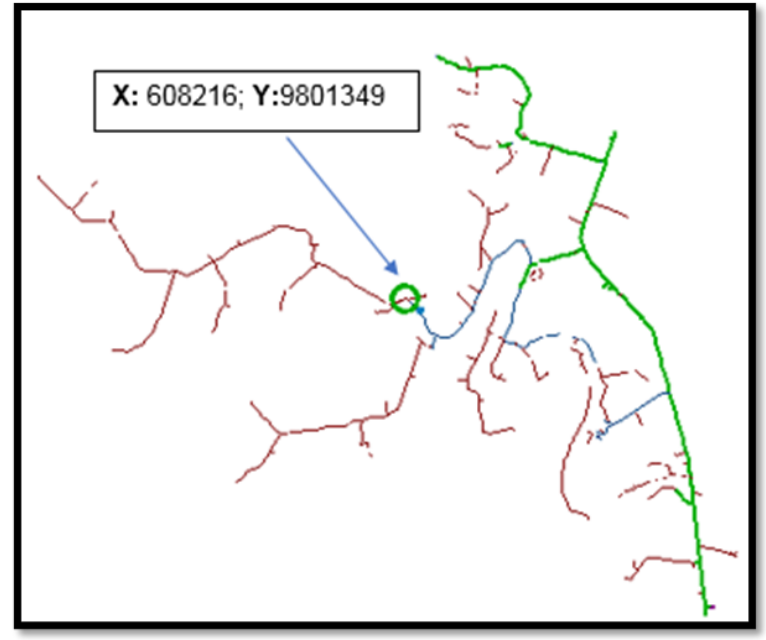

Figura 6. Ubicación de regulador monofásico automático. Software CYME

En la figura 7 y 8, se muestra el perfil de voltaje en demanda máxima y mínima del ramal monofásico de $8,67 \mathrm{~km}$ visto del arranque hasta el último tramo de línea con la operación de regulador monofásico automático.

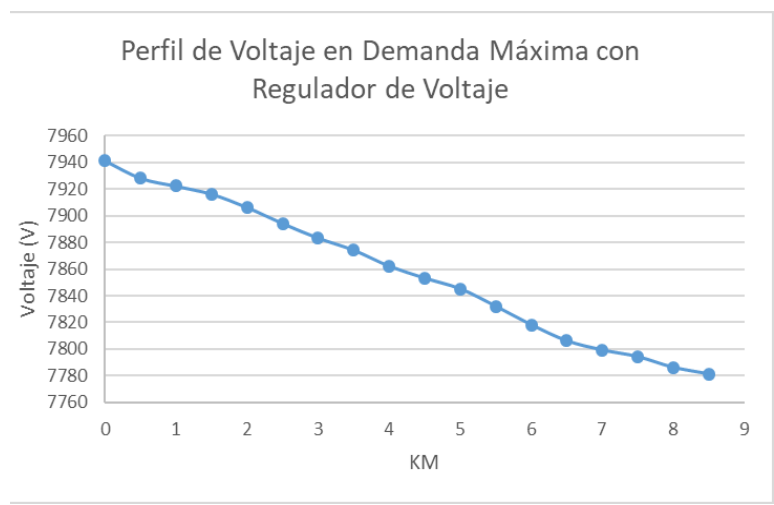

Figura 7. Perfil de voltaje en demanda máxima del ramal monofásico con regulador. Software CYME

Con la instalación del regulador, se verifica que los voltajes en demanda máxima a lo largo del ramal monofásico se encuentran en el rango de 7941-7781 (V) lo cual resulta un porcentaje de variación de voltaje en promedio de $-1,33 \%$ que está dentro del límite del $\pm 6 \%$ estipulado en la regulación del ARCONEL 005/18, entregando así, una buena calidad de servicio eléctrico y sin incurrir en sanciones a la distribuidora. 
Perfil de Voltaje en Demanda Mínima con Regulador de Voltaje

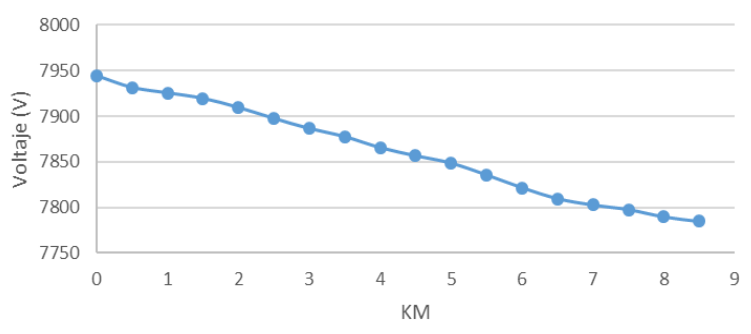

Figura 8. Perfil de voltaje en demanda mínima del ramal monofásico con regulador. Software CYME

Con la instalación del regulador, se verifica que los voltajes en demanda mínima a lo largo del ramal monofásico se encuentran en el rango de 7945-7785 (V) lo cual resulta un porcentaje de variación de voltaje en promedio de $-1,28 \%$ que está dentro del límite del $\pm 6 \%$ estipulado en la regulación del ARCONEL 005/18, entregando así, una buena calidad de servicio eléctrico y sin incurrir en sanciones a la distribuidora.

\section{Conclusiones}

El análisis se realizó en un ramal monofásico de un alimentador de distribución, en donde se plantearon dos escenarios: con demanda mínima y con demanda máxima, con el fin de considerar todas las problemáticas que se pudieran generar luego de mejorar los niveles de tensión en las redes de distribución. En demanda mínima se redujo el porcentaje de variación de voltaje promedio de $-5,12 \%$ a $-1,85 \%$, y en demanda máxima se redujo el porcentaje de variación de voltaje promedio de $-8,71 \%$ a -1,33\%.

El problema de caída de voltaje se origina cuando el consumo de energía aumenta en las horas pico (demanda máxima), lo que provoca que la variación de voltaje promedio sea de $-8,71 \%$ y que se exceda del límite que admite el ARCONEL.

La regulación del ARCONEL 005/18 exige a las empresas eléctricas de distribución un porcentaje de variación de voltaje, en media tensión, que no exceda del $\pm 6 \%$, caso contrario la distribuidora recibirá una sanción de 20 SBU (salario básico unificado) por cada índice incumplido. Con la instalación del regulador de voltaje en un nodo estratégico de la red monofásica, se notó una gran mejoría de los niveles de tensión tal como se demuestra en el punto 2 de la sección E del desarrollo de éste artículo, con lo cual se evita que la empresa distribuidora reciba algún tipo de sanción por dicho incumplimiento.

La solución con reguladores de voltaje es sólo para este caso puntual, ya que las subestaciones de distribución generalmente poseen de 2 a 5 alimentadores, los cuales también se ven afectados por un voltaje bajo en sus redes, por los problemas presentados en la sección $\mathrm{C}$ del desarrollo de éste artículo, los cuales son: sobrecarga, largas distancias, desbalance, calibre de conductor, bajo voltaje en el punto de entrega, etc.

Para los análisis realizados, siempre es indispensable que la distribuidora cuente con todo el equipamiento necesario como analizadores de redes instalados en la cabecera y en la mitad de la troncal del alimentador, así como mantener actualizada la topología de las redes del alimentador.

De acuerdo a los resultados obtenidos se puede comprobar que el uso de los reguladores Quick Drive Tap fue satisfactorio para sistemas de distribución monofásicos. La misma aplicación se la puede realizar en sistemas de distribución trifási$\cos [14]$.

Es recomendable que los controladores de los reguladores de voltaje sean integrados al sistema SCADA de la distribuidora para poder tener control del regulador, además de visualización de su comportamiento en la red instalada, para futuros análisis.

Además de la instalación de reguladores de voltaje, existen varios tipos de aplicaciones para la mejoría de los niveles de tensión en redes de distribución de medio voltaje. Éste caso en particular solo es aplicable en redes de media tensión, ya que los reguladores de voltaje solo se fabrican para soportar hasta los niveles de medio voltaje y son versátiles para ser instalados tanto en la cabecera como a lo largo del alimentador de la red de distribución. A nivel de distribución en alta tensión se recomiendan otro tipo de aplicaciones, como autotransformadores o la construcción de subestaciones cerca de la mayor concentración de carga. 


\section{Referencias}

[1] Castro, J. C., Lagos, G. S., \& Gonzalez, O. A. (2017). Simulation and measuring transients in On-Load Tap Changers. IEEE Latin America Transactions, 15(10), 1901-1907. https:// doi.org/10.1109/TLA.2017.8071234

[2] CONELEC. (2018). PROYECTO DE REGULACIÓN SOBRE CALIDAD DEL SERVICIO DE DISTRIBUCIÓN Y COMERCIALIZACIÓN DE ENERGÍA ELÉCTRICA INFORME DE SUSTENTO FINAL Dirección Nacional de Regulación Técnica Diciembre 2018. 20-30. www.regulacionelectrica.gob.ec

[3] Felber, L. A., Arango, H., Bonatto, B. D., \& Gouvêa, M. R. (2011). Voltage regulation in electric energy distribution substations. 2010 IEEE/PES Transmission and Distribution Conference and Exposition: Latin America, T and D-LA 2010, 846-852. https://doi. org/10.1109/TDC-LA.2010.5762983

[4] Katira, M. J., \& Porate, K. B. (2009). Computer simulation of 132 / $11 \mathrm{KV}$ distribution sub station using Static Var Compensator (SVC) for voltage enhancement - A case study. 2009 2nd International Conference on Emerging Trends in Engineering and Technology, ICETET 2009, 521-526. https://doi. org/10.1109/ICETET.2009.61

[5] Liu, J., Ge, H., Yang, Y., Guo, Q., Qian, F., Sun, H., Zhang, M., \& Wang, B. (n.d.). A Two-level Voltage Stability Monitoring $\mathrm{Me}$ thod. 1-5.

[6] Ortiz, A.; Jativa, J. . (2015). Análisis de Estabilidad de Voltaje en Estado Estable del Sistema de Subtransmisión de la Empresa Eléctrica Quito. Statewide Agricultural Land Use Baseline 2015, 1(Xc). https://doi. org/10.1017/CBO9781107415324.004

[7] Rios, J. P. B., Chirre, J. L. C., \& Jurado, R. C. (2017). Real time voltage stability supervision on a distribution network using phasor measurements without high voltage network data. 2017 IEEE Power and Energy
Conference at Illinois, PECI 2017. https://doi. org/10.1109/PECI.2017.7935762

[8] Sagara, M., Sediqi, M. M., Senjyu, T., Danish, M. S. S., \& Funabashi, T. (2017). Voltage stability improvement by optimal active power and reactive power output control of storage battery system. IEEE Region 10 Annual International Conference, Proceedings/TENCON, 2671-2674. https://doi.org/10.1109/TENCON.2016.7848523

[9] Vournas, C., Lambrou, C., Anagnostopoulos, I., Christoforidis, G., \& Kabouris, J. (2015). Distributed reactive support and voltage stability limits: The example of Peloponnese in the Hellenic Interconnected System. IEEE Power and Energy Society General Meeting, 2015-Septe, 1-5. https://doi.org/10.1109/ PESGM.2015.7286603

[10] Xiong, X., \& Wang, F. (2013). A new method for voltage stability evaluation of distribution network. Proceedings - 2013 2nd International Symposium on Instrumentation and Measurement, Sensor Network and Automation, IMSNA 2013, 1, 1054-1058. https://doi. org/10.1109/IMSNA.2013.6743462

[11] Zhang, B. Da, \& Guo, H. W. (2012). The control strategy for optimization of voltage and reactive power in substation based on load forecasting. 2012 Spring World Congress on Engineering and Technology, SCET 2012 Proceedings, 1-4. https://doi.org/10.1109/ SCET.2012.6342119

[12] Zhao, W. K., He, J. H., Bo, Z. Q., \& Klimek, A. (2009). The improvement of the digital differential relay in on-load tap changer transformer. 1st International Conference on Sustainable Power Generation and Supply, SUPERGEN '09, 1, 3-6. https://doi. org/10.1109/SUPERGEN.2009.5348004

[13] Cyme Internacional T\&D Inc. "Análisis básicos de Cymdist - Guia del usuario". Canada, 2011 
[14] Gallego, L., \& Padilha-Feltrin, A. "Voltage Regulator Modeling for the Three-Phase Power Flow in Distribution Networks". IEEE. 2008 